CW15

$5: 30 \mathrm{pm}$

\section{Ultrahigh bandwidth MSM traveling-wave} photodetectors

Jin-Wei Shi, Kian-Giap Gan, ${ }^{*}$ Yi-Jen Chiu, ${ }^{*}$ ChiKuang Sun, ${ }^{* *}$ Yin-Jay Yang, ${ }^{\dagger}$ John. E Bowers, ${ }^{\ddagger}$ Graduate Institute of Electro-Optical Engineering, National Taiwan University, Taipei, TAIWAN, R.O.C.; ${ }^{*}$ Department of Electrical and Computer Engineering University of California, Santa Barbara, CA 93106, USA; ** Graduate Institute of Electro-Optical Engineering and Department of Electrical Engineering, National Taiwan University, Taipei, TAIWAN, R.O.C.; E-mail: sun@cc.ee.ntu.edu.tw; ${ }^{\dagger}$ Department of Electrical Engineering, National Taiwan University, Taipei, TAIWAN, R.O.C.; ${ }^{\ddagger}$ Department of Electrical and Computer Engineering, University of California, Santa Barbara, CA 93106, USA

Traveling-wave photodetector (TWPD) $)^{1,2}$ attracts attentions due to its high bandwidth-efficiency product by replacing the traditional R-C bandwidth limitation by velocity mismatch between optical/electrical waves in a waveguide structure. A bandwidth-efficiency product of 76 $\mathrm{GHz}$ (with $180 \mathrm{GHz}$ bandwidth) at $800 \mathrm{~nm}$ wavelength was demonstrated with GaAs-based p-i-n TWPD structures. ${ }^{1}$ Recently a record bandwidth of $560 \mathrm{GHz}$ was achieved in the same wavelength regime using low-temperature-grown (LTG) GaAs as the photo-absorption layer ${ }^{2}$ of a $p-i-n$ TWPD, utilizing the sub-picosecond carrier trapping time of the LTG-GaAs at the expense of internal quantum efficiency. Even though high bandwidth performance was already demonstrated, due to the slow microwave propagation mode in a p-i-n structure, further bandwidth improvement was limited by the velocity mismatch bandwidth. In this paper we demonstrate a new type of ultra-high speed photodetector: MSM TWPD. By utilizing LTG-GaAs as the absorption layer, this device structure provides quasi-TEM microwave mode with a velocity close to or even higher than the optical velocity with an extremely wide velocity matched bandwidth. MSM TWPD structure has a better bandwidth performance than $\mathrm{p}$-i-n TWPD owing to its lower mi-
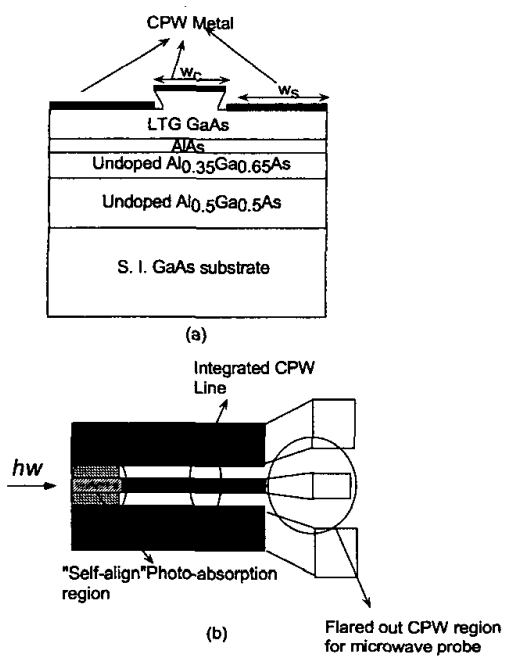

CWI5 Fig. 1. (a) Device cross sectional diagram and (b) top view. crowave loss, higher microwave velocity, and better impedance matching between the electrical transmission line of TWPDs and external electric load. With a preliminary device fabrication using self-aligned structures, the measured impulse response exhibits 0.8 ps pulsewidth with a corresponding $3 \mathrm{~dB}$ bandwidth over $570 \mathrm{GHz}$ even under high power excitation. Even wider bandwidth performance was obtained for low power excitation. These side-pumped MSM TWPDs also have a better efficiency $(>5 \%)$ compared with LTG-GaAs based MSM vertically-illuminated photodetectors. ${ }^{3}$ Without complex isolation process or E-beam lithography, this novel device is easily fabricated and offers a better way than $p$ $i-n$ TWPD or MSM VPD to be integrated as TWPD array for high power THz emitters.

The cross section and top view of the demonstrated MSM TWPD device are shown in Fig. 1. $3 \mu \mathrm{m}$-thick $\mathrm{Al}_{0.5} \mathrm{Ga}_{0.5}$ As layer is designed for optical isolation. $1 \mu \mathrm{m}$-thick $\mathrm{Al}_{0.35} \mathrm{Ga}_{0.65} \mathrm{As}$ and $500 \mathrm{~nm}$-thick LTG-GaAs are for waveguiding and photo-absorption purposes. Utilizing only semiinsulating LTG-GaAs and high resistive undoped semiconductor layers, this device has much lower microwave loss, higher microwave velocity, and better impedance matching between TWPDs and external electrical load or transmission line than p-i-n based TWPDs, implying that MSM-TWPD has a wider bandwidth than $\mathrm{p}-\mathbf{i}-\mathbf{n}$ based TWPD especially for long-length device with high saturation current. Three metal strips act as CPW to collect the photo-generated microwave signal. The center strip is naturally separated with ground planes by the depth and under-cut profile of etched ridge-mesa, and $200 \sim 300 \mathrm{~nm}$ distance between metal strips was easily achieved without complex E-beam lithography. This narrow-gap CPW can not only increase the responsivity of TWPD due to short carrier transport time but reduce microwave radiation loss when it operates near $\mathrm{THz}$ regime.
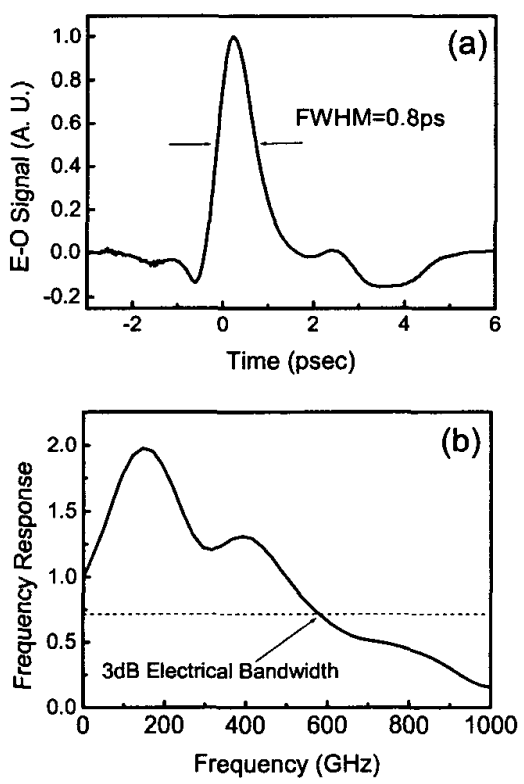

CWI5 Fig. 2. (a) EO response of a MSM TWPD under $800 \mathrm{~nm}$ excitation and (b) its corresponding Fourier transform.
Figure 2 shows an example of the device impulse response under $2.3 \mathrm{~mW}$ average power excitation with an $80 \mathrm{MHz}$ 150fs Ti:sapphire laser at $800 \mathrm{~nm} .0 .8 \mathrm{ps}$ pulsewidth with a transformed $3 \mathrm{~dB}$ bandwidth of $570 \mathrm{GHz}$ (fig. 2b) was obtained. With lower power excitation, pulsewidth will drop down to the order of 0.5 ps with an even higher $3 \mathrm{~dB}$ bandwidth. The total quantum efficiency is $\sim 5 \%$ under high bias. More bias-dependent and annealing-temperature-dependent performances will be discussed in our presentation.

\section{Reference}

1. K.S. Giboney, M.J.W. Rodwell, and J.E. Bowers, "Traveling-Wave Photodetector Design and Measurements," IEEE Journal Of Selected Topics In Quantum Electronics, 2, 622-629 (1996).

2. Y.J. Chiu, S.B. Fleischer, and J.E. Bowers, "High-Speed Low-Temperature-Grown GaAs p-i-n Traveling-Wave Photodetector," IEEE Photon. Technol. Lett. 10, 1012-1014 (1998).

3. S.Y Chou and M.Y. Liu, "Nanoscale TeraHertz Metal-Semiconductor-Metal Photodetectors," IEEE Journal of Quantum Electronics, 28, 2358-2368 (1992).

Ultrafast all-optical switching using a frequency shift accompanied by crossphase modulation in a semiconductor optical amplifiler

Shigeru Nakamura, Yoshiyasu Ueno, Kazuhito Tajima, System Devices and Fundamental Research, NEC Corporation, 34 Miyukigaoka, Tsukuba, Ibaraki 305-8501, Japan; E-mail: snakamura@dy.jp.nec.com

All-optical switches are quite attractive for optical demultiplexing and for other various optical signal processing in future optical-time-divisionmultiplexing (OTDM) networks. Optical demultiplexers will likely require switching operation for more than 100 -Gbps signals at a repetition rate of $10-40 \mathrm{GHz}$ with low control-light power.' A promising approach for such applications is a transient cross-phase modulation (T-XPM) alloptical switch, ${ }^{2,3}$ which features not only ultrafast switching with low control-light power but also a quite simple configuration. The simple configuration leads to many advantages such as compactness, stability, and polarization-insensitivity. In this report, we present ultrafast (corresponding to 168 -Gbps demultiplexing) and high-repetition $(10 \mathrm{GHz})$ operations of the T-XPM alloptical switch with a control-pulse energy of less than $1 \mathrm{pJ}$. These operations have been achieved by using a semiconductor optical amplifier (SOA) as a nonlinear waveguide and setting the control pulse duration shorter than the signal pulse duration.

The T-XPM all-optical switch is composed of a semiconductor nonlinear waveguide and a filter (Fig. 1(a)). In the nonlinear waveguide, an ultrashort control pulse excites a carrier density change and thus causes a nonlinear phase shift for the signal light. This nonlinear phase shift rapidly rises nearly within the control-pulse duration and then slowly relaxes. Ultrafast switching with the T-XPM switch is made possible by 